\title{
GRACE and loop integrals
}

\section{J. Fujimoto* and T. Kaneko,}

High Energy Accelerator Research Organization (KEK), 1-1 Oho Tsukuba, Ibaraki 305-0801, Japan

E-mail: junpei.fujimoto@kek.jp, toshiaki.kaneko@kek.jp

The multi-loop integral is an indispensable component for the perturbative calculation, because the high statistics and high energy collider-physics like the large hadron collider (LHC) and the international linear collider (ILC) requires the theoretical predictions with enough high-accuracy. The ring theory of differential operators and polynomials are applied to analyze the structure of the multi-loop integral. Using the symbolic manipulation system, $b$-functions and $P$-operators are constructed for some cases of the 2-loop diagrams.

"Loops and Legs in Quantum Field Theory" 11th DESY Workshop on Elementary Particle Physics April 15-20, 2012

Wernigerode, Germany

\footnotetext{
*Speaker.
} 


\section{Introduction}

High energy physics at the current and future colliders like the LHC and the ILC requires theorists to carry out large-scale calculations with multi-body productions. In the perturbative approach, the number of Feynman graphs to be calculated grows rapidly as the number of produced particles increases and as the order of perturbation becomes higher. For example, several tens of diagrams typically appear in the final four-body processes at the tree level, but a few thousand diagrams emerge in the 1-loop correction. Performing such computation is absolutely beyond the human power if it should be done by hand. Since the procedure of a perturbation calculation is well established, computers must be able to take the place of the human hand. In the past decade, several groups have developed computer programs which generate Feynman diagrams and calculate cross sections automatically. The GRACE $[1,2]$ system is such a program package for the automatic calculation of the amplitudes, based on the Feynman rules of the standard model (SM) and also of the minimal supersymmetric extension of the standard model (MSSM), which has also a capability to generate the higher order diagrams beyond the 1-loop level.

For simplicity we consider scalar loop integrals throughout the paper. The general expression of scalar loop integrals in Feynman parametric representation is

$$
(-1)^{N} \frac{\Gamma(N-\omega L / 2)}{(4 \pi)^{\omega L / 2}} \int_{0}^{1} \prod_{i=1}^{N} d x_{i} \delta\left(1-\sum_{i=1}^{N} x_{i}\right) \frac{C^{N-\omega(L+1) / 2}}{(D-i \varepsilon C)^{N-\omega L / 2}}
$$

where $L$ is the number of loops, $N$ is the number of internal particles and $\omega$ is the number of spacetime dimensions. Here, $C$ and $D$ are polynomials of the Feynman parameters $\left(x_{i}, i=1, \cdots, N\right)$ and they are determined by the topology of the corresponding diagram. An infinitesimal parameter, $\varepsilon$, is introduced to make the denominator non-zero throughout the integration domain.

However, the analytical expressions of the integration of the Eq.(1.1) are only known restrictedly except for the one-loop case[3]. In this paper, we discuss the application of the theory of the ring of differential operators with polynomial to analyze the structure of the multi-loop integral of Eq.(1.1).

\section{2. $b$-function and $P$-operator}

Let $f(x) \in K[x]=K\left[x_{1}, \cdots, x_{n}\right]$ be a polynomial of $n$ variables with coefficients in a field $K$ of characteristic zero. Let us denote $D_{n}(K):=K\left[x_{1}, \cdots, x_{n}\right]\left\langle\partial_{1}, \cdots, \partial_{n}\right\rangle$, the rings of differential operators with polynomial and formal power series coefficients respectively with $\partial_{i}=\partial / \partial x_{i}$ and $\partial=\left(\partial_{1}, \cdots, \partial_{n}\right) .\left(D_{n}(K)\right.$ is called the Weyl algebra over $K$.) Let $s$ be a parameter. Then the $b$ function (or the Bernstein-Sato polynomial) $b(s)$ associated with $f(x)$ is the monic polynomial of the least degree $b(s) \in K[s]$ satisfying

$$
P(s, x, \partial) f(x)^{s+1}=b(s) f(x)^{s}
$$

with some $P(s, x, \partial) \in D_{n}(K)[s]$, P-operators.

The $b$-function was introduced in the theory of prehomogeneous vector spaces by M. Sato in early1970s. Simultaneously and independently, the existence of $b(s)$ was proved by I. N. Bernstein in 1971[4]. The background of introduction of $b$-functions is related to the analytical continuation of the hyperfunctions $f(x)_{+}^{s}$. 


\section{Application of $b$-function to the loop integrals}

The application of this equation was first introduced by F. Tkachov[5] to one-loop integrals with the closed form as follows;.

$$
I=\int d(x) D(x)^{s}=\int d(x) \frac{1}{b(s)} P(s, x, \partial) D(x)^{s+1},
$$

where $D(x)$ is a quadratic form of $x=\left(x_{1}, \cdots, x_{n}\right)$,

$$
D(x)=x^{T} V x+2 R^{T} x+Z,
$$

with

$$
P=\frac{1}{\Delta}\left((1+s)-\frac{\left(x^{T}+A^{T}\right) \partial}{2}\right), \quad A=V^{-1} R, \quad \Delta=\left(Z-R^{T} V^{-1} R\right), \quad b(s)=(s+1),
$$

where $V$ is a $n \times n$ matrix and $R$ is a $n$ vector.

The extensive application of this equation and even the numerical application were discussed in Refs.[6, 7].

On the other hand, in the case of multi-loop integrals, the $P$-operators generally consist of higher derivatives on $x$. In order to avoid cumbersome studying of the linear system of equations to derive the $P$-operators, the papers [8], [9], [10] and [11], proposed to apply the functional relation Eq.(3.1) to the one-loop sub-diagram which has the largest number of internal lines in a 2-loop diagram. As the results, the integrand can be made smooth, apart from a factor $b(s)$ of Eq.(1.1) which is now a polynomial in $x_{s}$, the Feynman parameters needed for the complementary sub-diagram with the smallest number of internal lines. This is called "Minimal B-T(Bernstein-Tkachov) approach".

Minimal B-T approach works well for the numerical integration of the 2-loop integrals. Even though, $b$-functions themselves are still interesting in order to analyze the structure of the multiloop integrals. For example, it is known that all roots of the $b$-functions are negative rational number[12]. Furthermore, there exists an algorithm to compute $b$-functions and to construct $P$ operators by T. Oaku[13], using Gröbner bases for rings of differential operators.

\section{Outline of the algorithm by T. Oaku}

According to [13], we summarize the algorithm to determine $b$-functions and $P$-operators.

1. Find annihilation ideal $\mathrm{Ann}_{D}$ on $f^{s}$ for rings with differential operators such as

$$
\operatorname{Ann}_{D} f^{s}=\left\{P(s) \in D_{n}(K)[s] \mid P(s) f^{s}=0\right\} .
$$

2. Because of $b(s)-P(s) f \in \operatorname{Ann}_{D} f^{s}, b(s)$ should be an element of $B_{f}$, which is the intersection of $\operatorname{Ann}_{D} f^{s}+D_{n}(K)[s] f$ and $K[s]$,

$$
b(s) \in B_{f}=\left(\operatorname{Ann}_{D} f^{s}+D_{n}(K)[s] f\right) \cap K[s] .
$$


3. During getting $B_{f}, Q_{i}(s)$ and $P(s)$ can also be obtained as

$$
b(s)=Q_{1}(s) P_{1}(s)+\cdots+Q_{k}(s) P_{k}(s)+P(s) f,
$$

where $P_{i}(s)$ is a generator of $\mathrm{Ann}_{D} f^{s}$.

4. Because of $P_{j} f^{s}=0$, this $P(s)$ satisfies $P(s) f^{s+1}=b(s) f^{s}$.

It is necessary to use the symbolic manipulation system to realize the above algorithm. The system should equip with the Gröbner bases engine and also can treat the Wyle algebra, $\left[\partial_{i}, x_{j}\right]=\delta_{i j}$ like Maple[14], Singular[15], Macaulay2[16], etc. Here we have used Risa/Asir [17] and OpenXM (Kan/Sm1) [18].

\section{Example for 2-loop non-planar vertex diagrams}

We have examined to apply the symbolic manipulation system to construct $b$-functions and $P$-operators to the 2-loop diagrams. Here we only show the results for some cases of 2-loop nonplanar vertex diagrams depicted in Fig.1. The corresponding Feynman parameter integral is as follows;

$$
I=\int d x_{1} \cdots d x_{6} \delta\left(1-\sum_{i=1}^{6} x_{i}\right) \frac{1}{D^{2}}
$$

where

$$
\begin{gathered}
D=f_{1} p_{1}^{2}+f_{2} p_{2}^{2}+f_{3} p_{3}^{2}-C \sum_{j} x_{j} m_{j}^{2}, \\
C=\left(x_{1}+x_{2}\right)\left(x_{3}+x_{4}+x_{5}+x_{6}\right)+\left(x_{3}+x_{4}\right)\left(x_{5}+x_{6}\right),
\end{gathered}
$$

and

$$
\begin{aligned}
& f_{1}=\left(x_{1}+x_{2}+x_{5}+x_{6}\right) x_{3} x_{4}+x_{1} x_{3} x_{6}+x_{2} x_{4} x_{5}, \\
& f_{2}=\left(x_{1}+x_{2}+x_{3}+x_{4}\right) x_{5} x_{6}+x_{2} x_{3} x_{6}+x_{1} x_{4} x_{5}, \\
& f_{3}=\left(x_{3}+x_{4}+x_{5}+x_{6}\right) x_{1} x_{2}+x_{2} x_{4} x_{6}+x_{1} x_{3} x_{5} .
\end{aligned}
$$

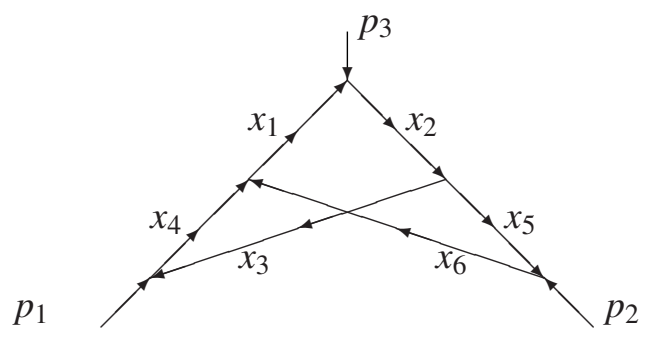

Figure 1: 2-loop non-planar vertex diagram.

After we set kinematical values of $p_{i}$ and $m_{i}$ as $p_{1}^{2}=p_{3}^{2}=10, p_{2}^{2}=0, m_{1}^{2}=1, m_{2}^{2}=2, m_{3}^{2}=3$, $m_{4}^{2}=4, m_{5}^{2}=5$ and $m_{6}^{2}=6$, and $x_{6}$ was eliminated with the $\delta$ function, the symbolic manipulation was applied, then we find the $b$-function for this $D$ as

$$
b(s)=(s+1)(2 s+3),
$$


which means the $P$-operator consists from the second derivatives at most, because $b$-function is represented in the quadratic form on $s$.

Actually the $P$-operator for this $D$ has the following form;

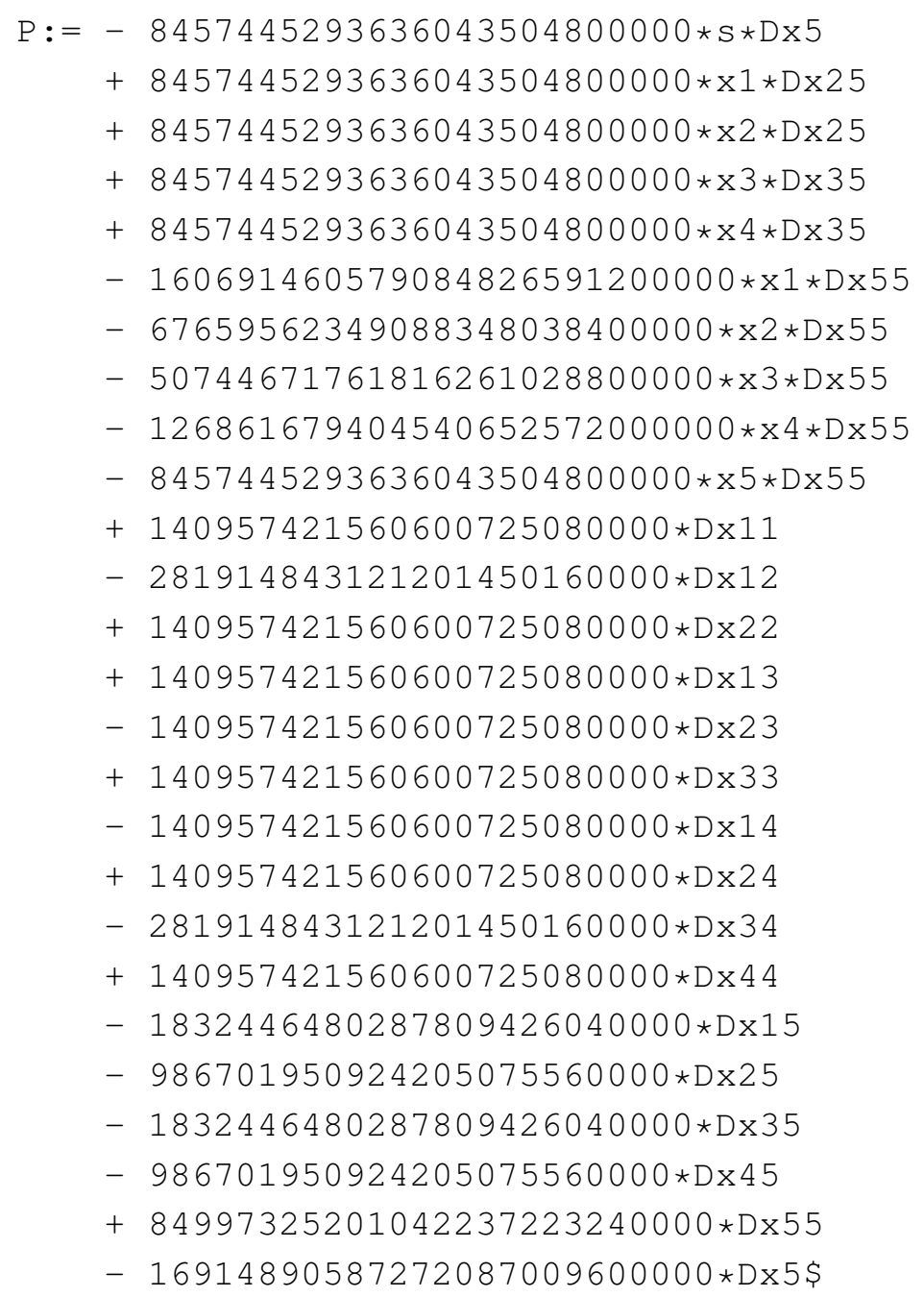

where differential operators are defined as $\mathrm{D} \times 5=\partial_{5}, \mathrm{D} \times 25=\partial_{2} \partial_{5}, \mathrm{D} \times 55=\left(\partial_{5}\right)^{2}$, and so on.

\section{Summary}

The $b$-functions might be useful to analyze the structure of the Feynman multi-loop integrals. There exists a general algorithm to derive $b$-functions and $P$-operators by T. Oaku. We have examined to construct $b$-functions and $P$-operators for some cases of 2-loop diagrams using symbolicsystems which treat the Gröbner bases engine with Wyle algebra. Application of $P$-operators to the numerical integration of the multi-loop integrals are still open questions.

\section{Acknowledgments}

Authors would like to thank Prof. Y. Shimizu for insightful discussions and his proof reading of the mamuscript. They also would like to thank Drs. T. Ishikawa, M. Jimbo, K. Kato, T. Kon, Y. 
Kurihara and F. Yuasa for their showing interest in this subject and continuous support. This work was supported in part by the Grant-in-Aid (No.20340063, No.23540328 and No.24540292) of JSPS, and by the CPIS program of Sokendai.

\section{References}

[1] Minami-Tateya Group, T. Ishikawa, et al., KEK-92-19 (1993).

[2] F. Yuasa, et al., Prog. Theor. Phys. Suppl. 138 (2000) 18; http://minami-home.kek.jp/ .

[3] G. 't Hooft and M. Veltman, Nuclear Physics B153 (1979) 365.

[4] I. N. Bernstein, Functional Anal. Appl. 5(1971) 89.

[5] F.V. Tkachov, Nucl. Instr. Meth. Phys. Res. A389 (1996) 309.

[6] G. Passarino, Nucl. Phys. B619(2001)257.

[7] A. Ferroglia, G. Passarino, M. Passera, S. Uccirati, Nucl. Phys. B650(2003)162.

[8] G. Passarino, S. Uccirati, Nucl. Phys. B629 (2002) 97.

[9] A. Ferroglia, G. Passarino, M. Passera, S. Uccirati, Nucl. Phys. B680 (2004) 199.

[10] S. Actis, A. Ferroglia, G. Passarino, M. Passera, S. Uccirati, Nucl. Phys. B703 (2004) 3.

[11] G. Passarino, C. Sturm, S. Uccirati, Phys. Lett. B655(2007)298.

[12] M. Kashiwara, Invent. Math. vol. 1016 (1976) 33.

[13] T. Oaku, Journal of Pure and Applied Algebra 117\&118(1997)495,

T. Oaku, Advances in Applied Mathematics 19(1997)61,

T. Oaku, Duke Math. J. 87(1997) 1.

[14] http://www.maplesoft.com/products/maple/

[15] http://www.singular.uni-kl.de/

[16] http://www.math.uiuc.edu/Macaulay2/

[17] M. Noro, http://www.math.kobe-u.ac.jp/Asir/

[18] N. Takayama, http://www.math.kobe-u.ac.jp/OpenXM/ 